\title{
Movable collimator system for SuperKEKB
}

\author{
T. Ishibashi®, S. Terui, Y. Suetsugu, K. Watanabe, and M. Shirai \\ High Energy Accelerator Research Organization (KEK), 305-0801, Tsukuba, Japan
}

(Received 3 March 2020; accepted 6 May 2020; published 13 May 2020)

\begin{abstract}
Movable collimators for the SuperKEKB main ring, which is a two-ring collider consisting of a $4 \mathrm{GeV}$ positron and $7 \mathrm{GeV}$ electron storage ring, were designed to fit an antechamber scheme in the beam pipes, to suppress background noise in a particle detector complex named Belle II, and to avoid quenches, derived from stray particles, in the superconducting final focusing magnets. We developed horizontal and vertical collimators having a pair of horizontally or vertically opposed movable jaws with radiofrequency shields. The collimators have a large structure that protrudes into the circulating beam and a large impedance. Therefore, we estimated the impedance using an electromagnetic field simulator, and the impedance of the newly developed collimators is lower compared with that of our conventional ones because a part of each movable jaw is placed inside the antechamber structure. Ten horizontal collimators and three vertical collimators were installed in total, and we installed them in the rings taking the phase advance to the final focusing magnets into consideration. The system generally functioned, as expected, at a stored beam current of up to approximately $1 \mathrm{~A}$. In high-current operations at $500 \mathrm{~mA}$ or above, the jaws were occasionally damaged by hitting abnormal beams, and we estimated the temperature rise for the collimator's materials using a Monte Carlo simulation code. In order to avoid damage to the jaw, we have started developing a collimator with low atomic number materials.
\end{abstract}

DOI: 10.1103/PhysRevAccelBeams.23.053501

\section{INTRODUCTION}

SuperKEKB, which is an upgrade project of KEKB, is an electron-positron collider aiming at extremely high luminosity [1]. The SuperKEKB main ring consists of two rings, a $7 \mathrm{GeV}$ electron ring termed the high energy ring (HER) and $4 \mathrm{GeV}$ positron ring termed the low energy ring (LER). The designed luminosity is $8 \times 10^{35} \mathrm{~cm}^{-2} \mathrm{~s}^{-1}$, and the beam currents are 2.6 and 3.6 A in the HER and LER, respectively. In the upgrade, approximately $93 \%$ of the LER, and $18 \%$ of the HER, vacuum components were replaced with new ones. The standard beam pipes in the HER Tsukuba straight section and LER have a beam channel and two antechambers. The inner and outer antechambers in the rings respectively work as a pumping channel for distributed pumping with a strip-type nonevaporable getter (NEG) pump and as a synchrotron radiation (SR) channel for reduction of the SR power density [2].

We previously designed new-type collimators to fit the antechamber scheme. The collimator is one of the vacuum components used to shield the non-Gaussian tail in bunches

Published by the American Physical Society under the terms of the Creative Commons Attribution 4.0 International license. Further distribution of this work must maintain attribution to the author(s) and the published article's title, journal citation, and DOI. by bringing heavy metal blocks in close proximity to the circulating beam. The collimator is used to suppress background noise in Belle II, which is a particle detector complex in SuperKEKB [3]. The collimators also function as machine protection systems by limiting physical apertures locally in the rings, and also prevent quenches in the superconducting final focusing magnets (QCS [4]) derived from issues such as unexpected beam loss.

There are two types of collimators: horizontal and vertical. The former and latter have a pair of horizontally and vertically opposed movable jaws, which generally comprise blocks manufactured from metals, as shown in Figs. 1(a) and 1(b), respectively.

The location of the collimators in the early stage of phase-3, which refers to the third commissioning stage of SuperKEKB that began in Spring 2019, for high energy physics experiments operating in the full detector configuration in Belle II [5], is shown in Fig. 2. (Strictly speaking, the present detector is not fully configured because only one of the two layers in the pixel detector has been installed. The full configuration is expected to be installed after 2021) In the HER, eight horizontal and eight vertical collimators each for the two arc sections were reused. These collimators were manufactured in 1998 and have been used for KEKB; they possess the standard racetrack shape in the HER arc sections with a height of $50 \mathrm{~mm}$ and full width of $104 \mathrm{~mm}$ [6]. The other collimators were newly designed and have continued to be developed since 2014 as commissioning progresses. These SuperKEKB-type 


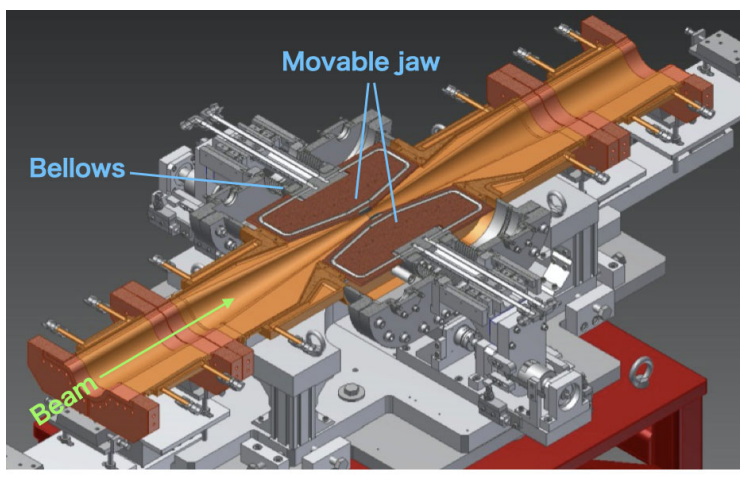

(a)

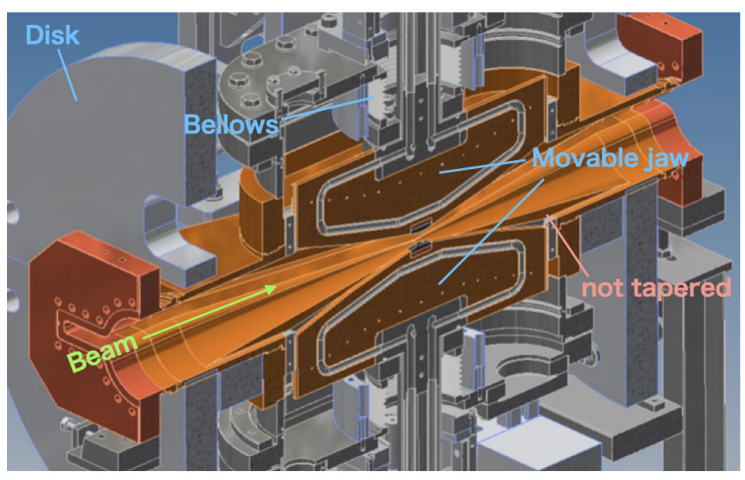

(b)

FIG. 1. Structure of (a) horizontal collimator and (b) vertical collimator.

collimators were installed in two arc sections in the LER and the Tsukuba straight section in both rings, as shown in Fig. 2.

The location from the viewpoint of the beam optics is shown in Fig. 3. The collimators are dispersed in the phase

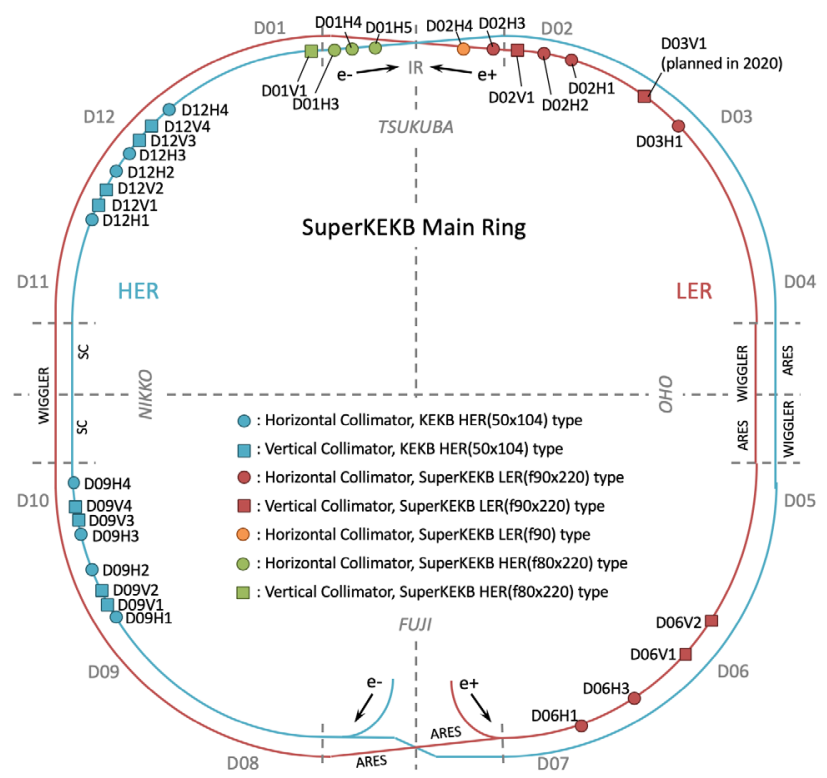

FIG. 2. Location of collimators in the SuperKEKB main ring. $\mathrm{H}$ and $\mathrm{V}$ in the collimator names refer to the horizontal and vertical collimators.

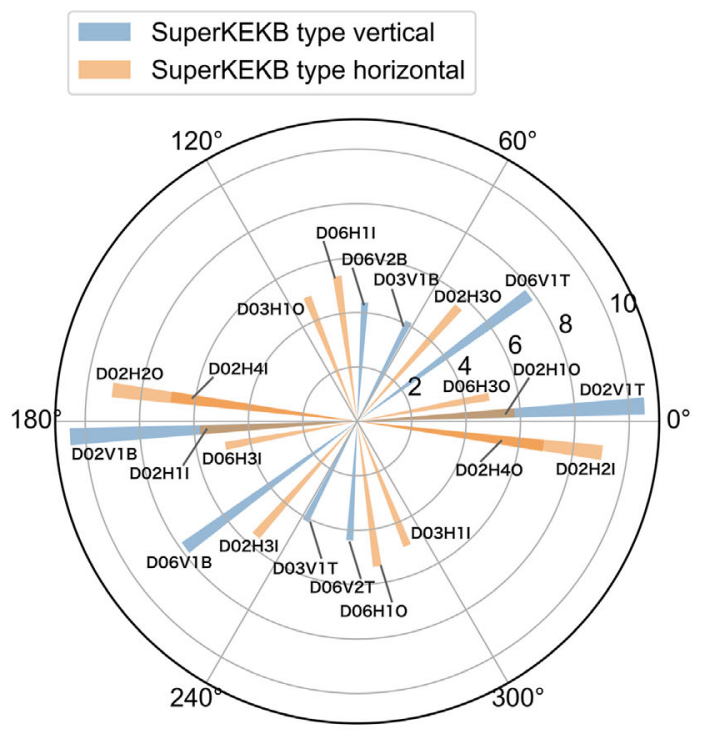

(a)

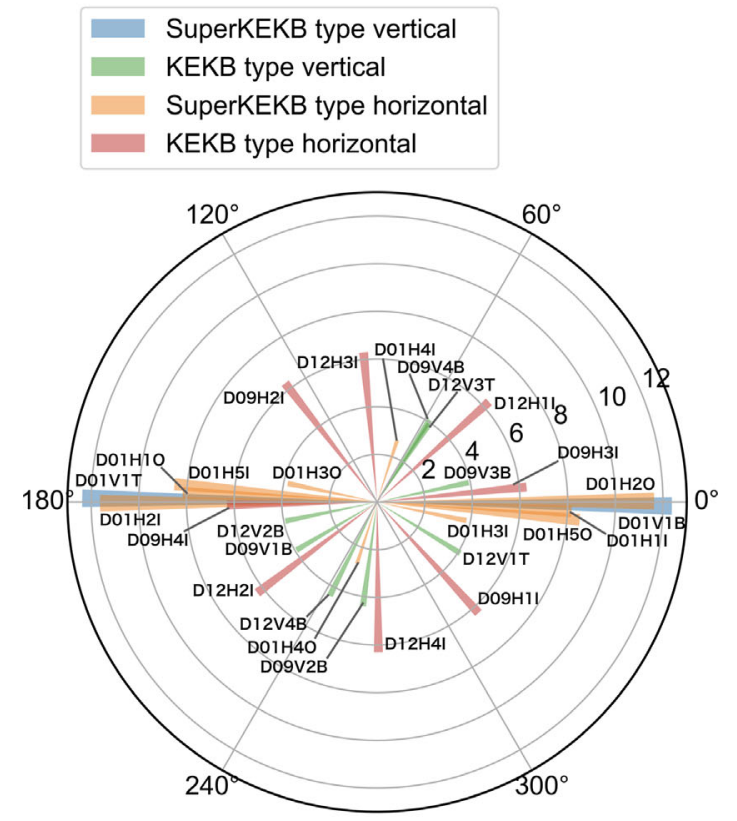

(b)

FIG. 3. Location of collimators in phases of the betatron oscillation for (a) LER and (b) HER. The angular coordinates refer to the phase advance to QC1RP in LER or QC1LE in HER, which are the closest final focusing magnets to IP; 0 and $180^{\circ}$ refer to the phase in the QC1RP or QC1LE. The radial coordinates refer to the square root of the horizontal or vertical beta function at each horizontal or vertical collimator (unit: $\mathrm{m}^{1 / 2}$ ), respectively. $\mathrm{T}, \mathrm{B}, \mathrm{I}$, or $\mathrm{O}$ in the collimator names refer to the location of the jaw (top, bottom, inner, or outer side in the main ring, respectively).

of the betatron oscillation as much as possible to cut off stray particles at the different phases. The KEKB-type collimators in the HER have a jaw on one side that can induce a bunch tilt to the beam traveling direction owing to 
the transverse wakefield and degenerate luminosity [7]. Therefore, these collimators were placed with a phase difference of approximately $180^{\circ}$ to cancel the transverse kick and relax the bunch tilt effect. For instance, the phases of the $\mathrm{D} 12 \mathrm{H} 1$ and $\mathrm{D} 12 \mathrm{H} 2$ collimators are approximately $42^{\circ}$ and $217^{\circ}$, respectively; thus, the difference is $175^{\circ}$ [see Fig. 3(b)]. Note that the SuperKEKB-type collimators have two opposite jaws in a chamber; therefore, the transverse kick is almost canceled within the collimators. Then, some of the SuperKEKB-type collimators were positioned at low beta functions as we could relax the bunch current threshold owing to the transverse mode coupled instability (TMCI) [8].

\section{DESIGN}

\section{A. Structure}

The cross section at the end of the collimator chamber, which is equipped with a pair of movable jaws, is the same as that of the beam pipes on the upstream or downstream. The end of the collimator is equipped with one of three types of cross sections: $f 90 \times 220, f 80 \times 220$, and $f 90$. The $\mathrm{f} 90 \times 220$ and $\mathrm{f} 80 \times 220$ types have a half width of $110 \mathrm{~mm}$ in the horizontal plane, which is the distance between the center of the beam channel and the inner wall of the antechamber; the prefix $f$ stands for the diameter of the beam channel, and the $\mathrm{f} 90 \times 220$ and $\mathrm{f} 80 \times 220$ types have 90 and $80 \mathrm{~mm}$ in the diameter, respectively. The $f 90$ type is a circular beam pipe without antechambers, having a diameter of $90 \mathrm{~mm}$. The collimator chamber consists of horizontally two halved chambers attached to a vacuum brazing, and the bonding plane is offset from the beam level by a few millimeters to prevent SR irradiation on the bonding plane. The chamber and jaws are made of oxygenfree copper as the main component. The MatsumotoOhtsuka (MO)-type flanges [9] and the racetrack shaped flanges, into which the jaw is inserted, are made of chromium copper and stainless steel, respectively. The collimator chamber has viewports; the viewports are sapphire windows and are used to check the condition of the tip of the jaw from the atmospheric side.

It is assumed that the collimator is operated with a distance of 0.4-25 $\mathrm{mm}$ between the center of the beam channel and the tip of the jaw (i.e., half aperture) for the vertical collimators and 2-30 $\mathrm{mm}$ for the horizontal collimators. The length and thickness of the jaw are 360 and $12 \mathrm{~mm}$, respectively; the jaw can be replaced with a new one when the tip is damaged, for instance. However, replacement is too difficult for the bottom jaw in the vertical collimator when the collimator is in the installed position. Therefore, two rotatable circular disks are attached to the outer side of the collimator chamber in the vertical type [see Fig. 1(b)]. The jaw has an internal cooling channel, which refers to a stainless-steel pipe with an outer diameter of $10 \mathrm{~mm}$, and is equipped with a tungsten block at the tip to shield stray particles, considering the high melting point of the construction material of the jaw and the short radiation length (RL) of $3.5 \mathrm{~mm}$. The longitudinal length of the tungsten block is $10 \mathrm{~mm}$, which corresponds to approximately 2.9 RL. These components are jointed with a hot isostatic press. Because there is a cavity structure behind the jaw when viewed from the beam channel, a contact finger-type rf shield is attached to the top and bottom of each jaw to prevent the intrusion of higher order modes (HOMs), as shown in Fig. 4. The rf fingers are made of silver-plated INCONEL. The contact surface on the chamber is made of rhodium-plated stainless steel. A contactless comb-type rf shield, which is also used in the bellows chambers in SuperKEKB [10], was positioned between the longitudinal end of the jaw and the facing surface on the chamber because the contact fingers on the longitudinal end break easily when the jaw is inserted into the chamber. This comb-type rf shield prevents the generation of dust, as dust may be a problem for the top-side jaw of the vertical collimators, especially because this dust may fall directly onto the circulating beam.

The collimators have a large structure that protrudes into the circulating beam, which may be a main source of impedance in the rings. In order to reduce the impedance, the angle of the slope in the collimator, which approaches the beam, is conventionally minimized to reduce the impedance [11]. However, the total length is increased in this scheme, and the spaces in which the systems can be installed are limited. In order to relax this limitation, a part of each movable jaw of the horizontal collimator is placed inside the antechamber. In the vertical collimator or the $f 90$ type horizontal collimator, the region in which the movable jaw is placed (14 mm in width), is not tapered, as shown in Fig. 1(b); thus, the part of the jaw is also hidden from the beam channel, regardless of whether an antechamber is present or not. For example, the angles of the slope in the SuperKEKB-type vertical collimators and in the KEKBtype vertical collimators are approximately $16^{\circ}$ and $13^{\circ}$, and the impedance in the former is less than that in the latter, as described below. Then, the total length of the SuperKEKBtype collimators and the set of KEKB-type collimators is 800 and $2000 \mathrm{~mm}$.

The collimators in PEP-II of SLAC were used as a reference for the basic design of the SuperKEKB type collimators [12]. The collimator has two opposite jaws with a contact finger-type if shield in the vacuum chamber and was operated up to approximately $3 \mathrm{~A}$ in the LER and $2 \mathrm{~A}$ in the HER of PEP-II [13].

\section{B. rf properties}

The effectivity of these rf shields and excitation of trapped modes were estimated by using Eigenmode Solver in CST STUDIO [14]. The simulation model and an example of a resonance mode are shown in Fig. 5. The frequency of the fundamental mode in the beam channel is 


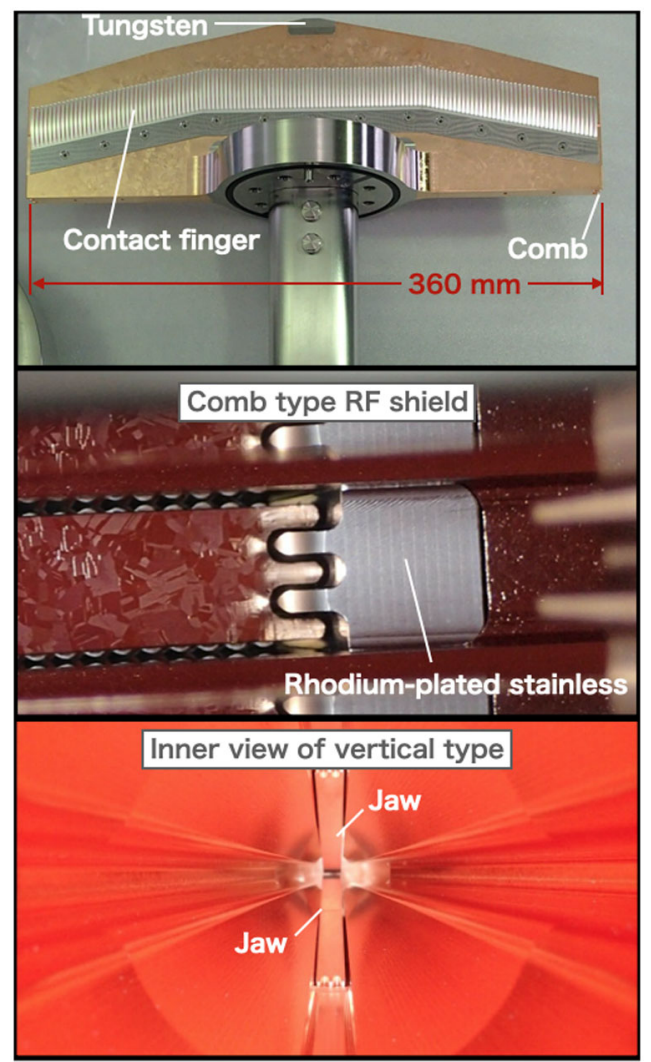

FIG. 4. Photograph of a jaw with rf shields.

$1.9557 \mathrm{GHz}$, and the direction of the electric field is horizontal, which refers to the $\mathrm{x}$-direction and $\mathrm{TE}_{111, x}$ mode. The resonance frequency of the next mode is $1.9849 \mathrm{GHz}$, and the direction of the electrical field is
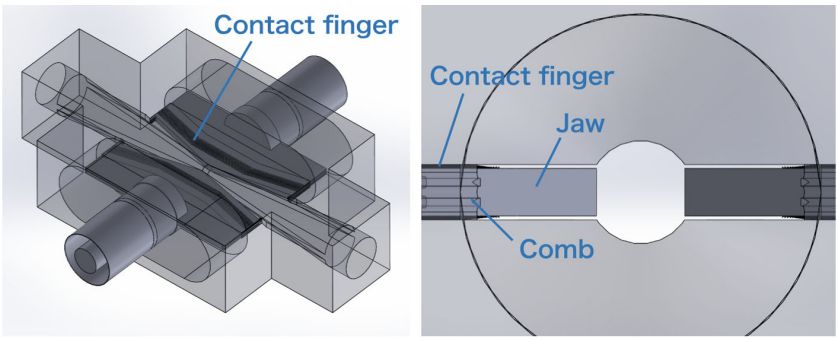

(a)

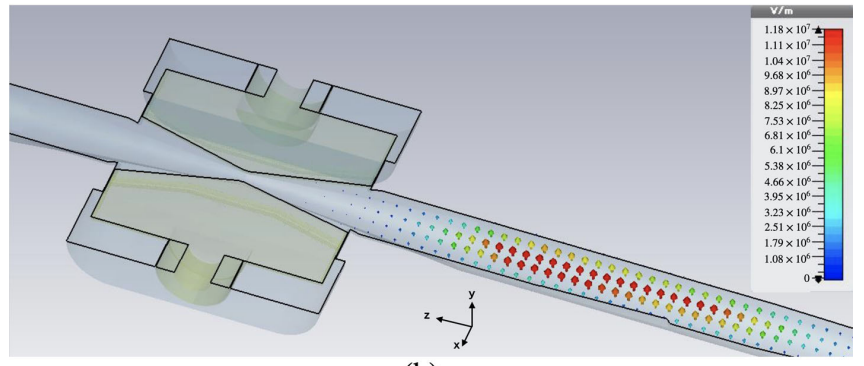

(b)

FIG. 5. (a) Simulation model and (b) electric field strength in $\mathrm{TE}_{111, y}$ mode $(1.9849 \mathrm{GHz})$, calculated with CST STUDIO. vertical, which refers to the $\mathrm{y}$-direction and $\mathrm{TE}_{111, y}$ mode. The "oozing" of the electric field from the beam channel to the cavity behind the jaw in the $\mathrm{TE}_{111, y}$ mode is larger than that in the $\mathrm{TE}_{111, x}$ mode, and the maximum electric field strengths in the beam channel and in the gap between the jaw and the chamber wall are $1.2 \times 10^{7}$ and $10 \mathrm{~V} \mathrm{~m}^{-1}$, respectively. Thus, the coupling between the beam channel and the cavity is 6 orders of magnitude smaller, and the $\mathrm{rf}$ shields around the jaw are sufficiently efficient in not only these modes but also the higher order modes.

The beam channel of the collimators is tapered to the center of the chamber to avoid excitation of the trapped modes around the jaws. For example, the diameter of the beam channel is narrowed from 90 to $27 \mathrm{~mm}$ at the center of the collimator in the $\mathrm{f} 90 \times 220$ type. The trapped mode is excited around the jaws in the vertical collimator with the straight shaped antechambers, as shown in Fig 6(a). This trapped mode is $\mathrm{TE}_{111, y}$, and the magnetic field rotates around the two jaws. Excitation of the trapped mode is

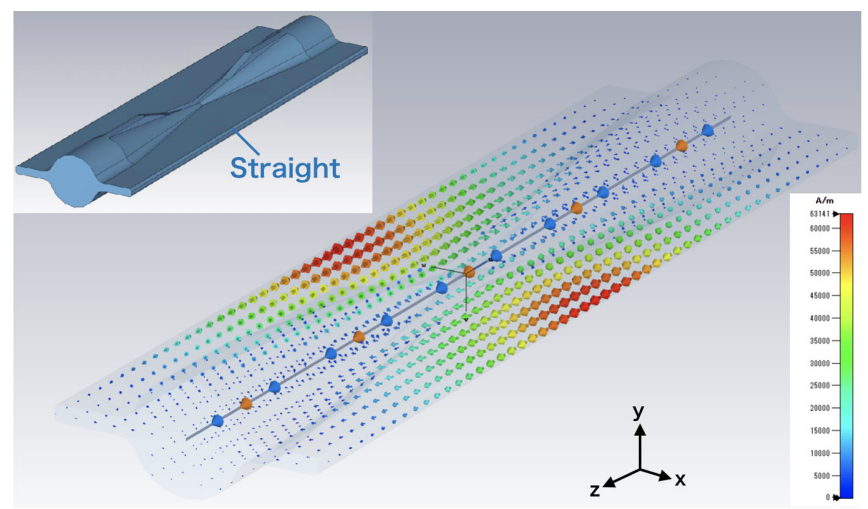

(a)

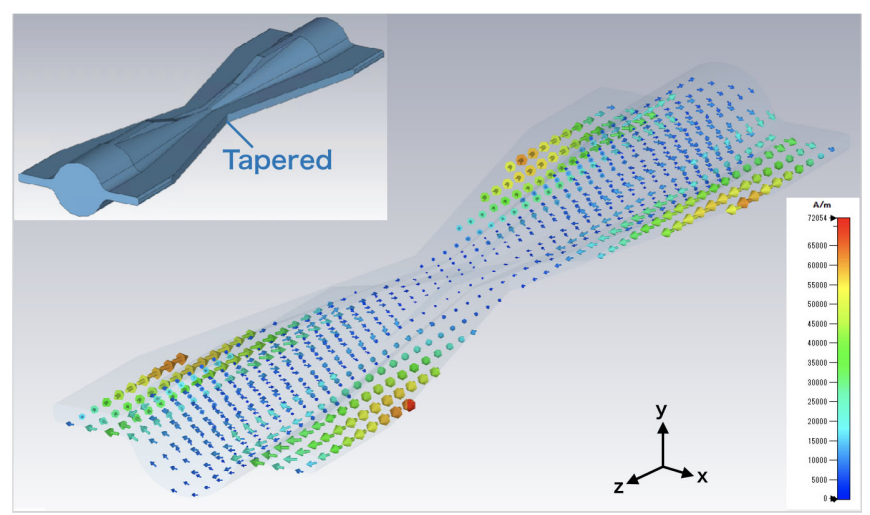

(b)

FIG. 6. Simulation model and magnetic field strength in (a) straight and (b) tapered antechambers, calculated with CST STUDIO. The tapered height at the center of the collimator is $70 \mathrm{~mm}$. The mode and the resonance frequency are $\mathrm{TE}_{111, y}$ and $0.7536 \mathrm{GHz}$ in the straight shape, $\mathrm{TE}_{112, y}$ and $1.1683 \mathrm{GHz}$ in the tapered shape, respectively. 


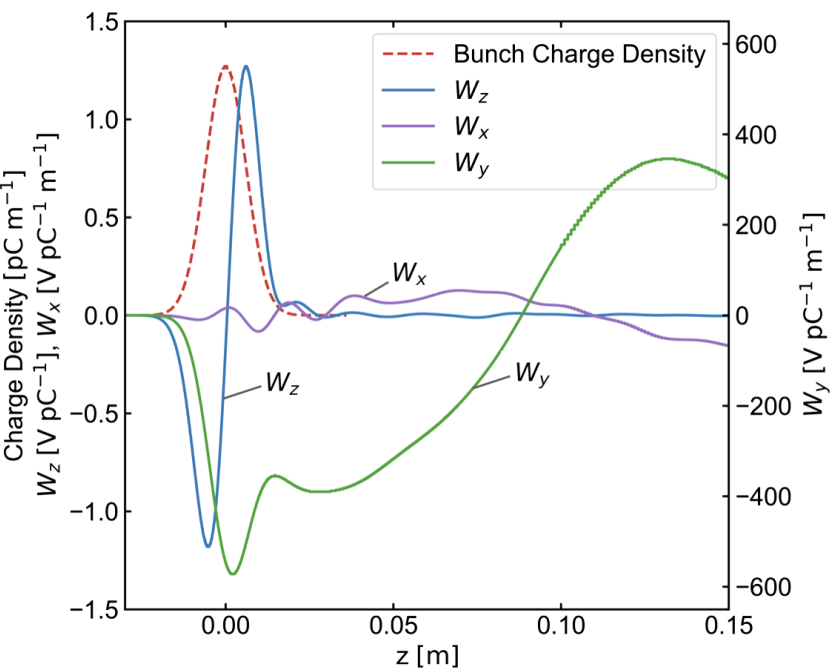

FIG. 7. Example of bunch profile, longitudinal $\left(W_{z}\right)$ and transverse $\left(W_{x}, W_{y}\right)$ wake potentials in SuperKEKB LER $(f 90 \times 220)$ type vertical collimator, calculated with GDFIDL (half aperture: $2 \mathrm{~mm}$ ). The bunch length and the beam offset from the center to the vertical or horizontal direction are 6 and $0.5 \mathrm{~mm}$, respectively.

prevented by tapering the inside of the antechambers to the center of the vertical collimator, as shown in Fig. 6(b).

\section{Impedance}

The impedance of the collimators was estimated using GDFIDL [15]. A set of the KEKB-type collimators consists of one movable jaw formed from a curved chamber and each has two bellows chambers at the upstream and downstream [6]; the chamber itself approaches the circulating beam. The total length of the collimator, including the four bellows chambers, is approximately $2000 \mathrm{~mm}$. In the KEKB-type collimator, the tip is made of pure titanium, and the longitudinal length is $40 \mathrm{~mm}$, which corresponds to approximately $1.1 \mathrm{RL}$.

Figure 7 presents an example of the bunch profile and longitudinal and transverse wake potentials in the vertical collimator having the $f 90 \times 220$ type cross section in the SuperKEKB LER. The bunch length (the standard deviation of the Gaussian-distributed beam in the longitudinal direction) used in this simulation is $6 \mathrm{~mm}$, which corresponds to the design value for SuperKEKB LER. The half aperture, which refers to the distance between the center of the beam channel and the tip of the jaw, is $2 \mathrm{~mm}$ ( $4 \mathrm{~mm}$ in the full aperture). The shape of the longitudinal wake potential corresponds to that of an inductive impedance source.

Figure 8 shows the loss and kick factors in a bunch length of $6 \mathrm{~mm}$. The loss and kick factors of the SuperKEKB collimators are less than those of the KEKB collimators, except for the loss factors when the half aperture is $15 \mathrm{~mm}$ or wider for the horizontal

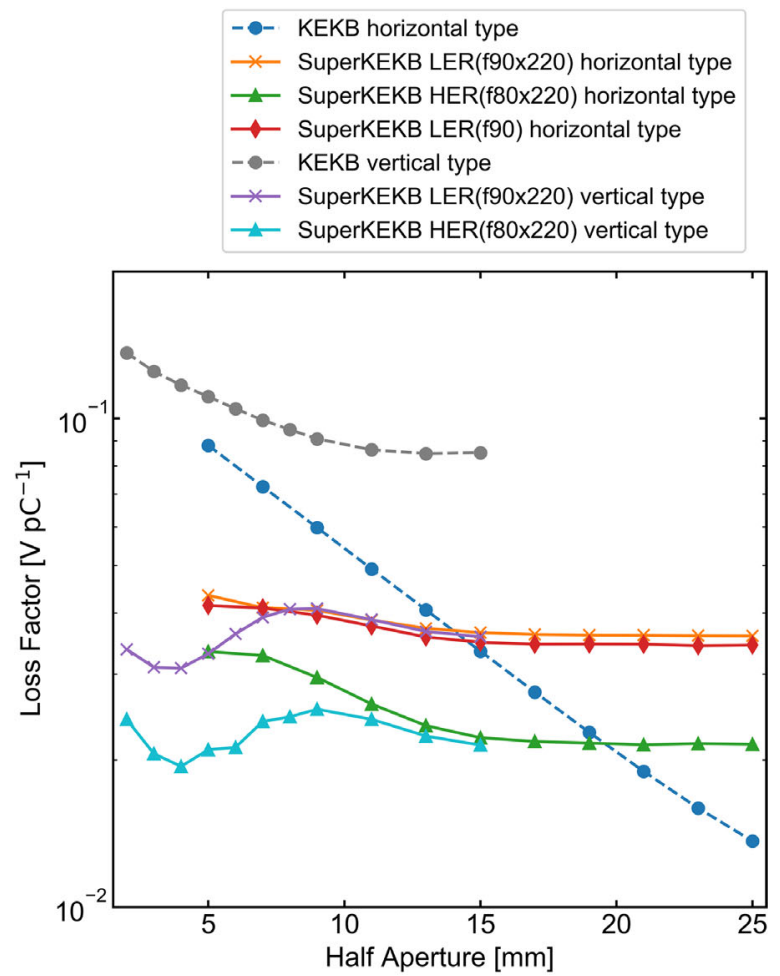

(a)

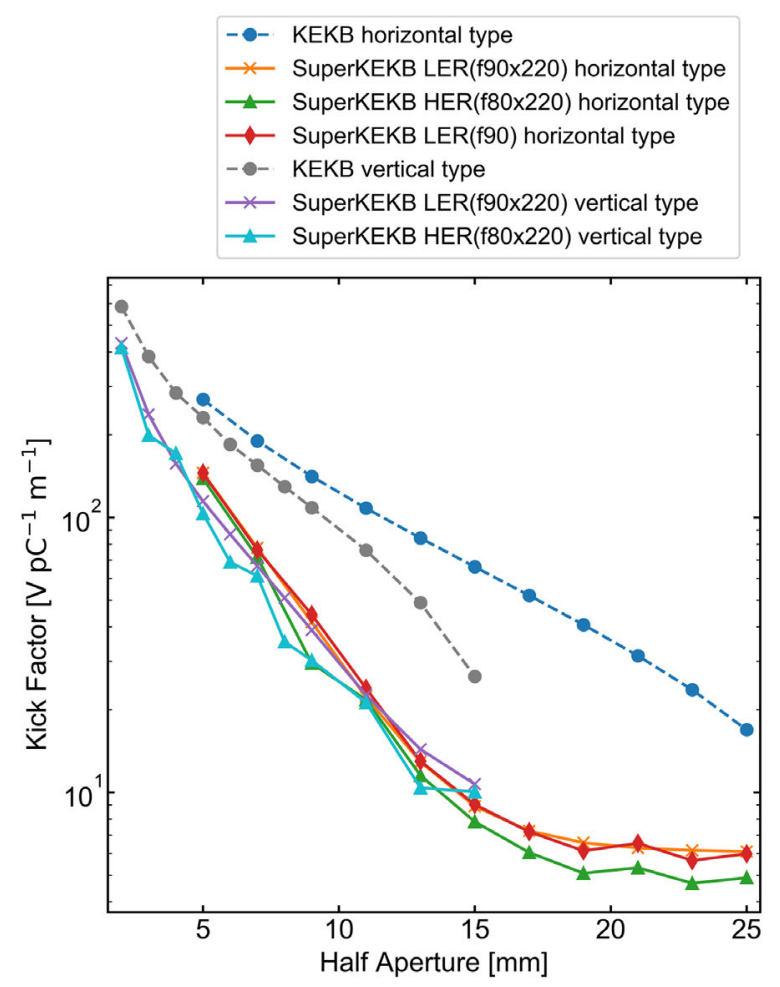

(b)

FIG. 8. (a) Loss and (b) kick factors for collimators as a function of the half apertures calculated with GDFIDL. The bunch length is $6 \mathrm{~mm}$. The kick factors are calculated with a beam offset of $1 \mathrm{~mm}$ from the center in the horizontal direction for the horizontal collimators or $0.5 \mathrm{~mm}$ in the vertical direction for the vertical ones. 
TABLE I. Estimation of loss factor $\left(k_{z}\right)$ in SuperKEKB LER, calculated with GDFIDL (bunch length: $6 \mathrm{~mm}$ ). The half apertures in the horizontal and vertical collimator are set to 2 and $5 \mathrm{~mm}$, respectively. The loss factor of the connection part of the MO flange is calculated by assuming a protrusion of $0.2 \mathrm{~mm}$ into the beam channel, with a width of $1 \mathrm{~mm}$ in the gasket [18].

\begin{tabular}{|c|c|c|c|}
\hline Component & $k_{z}\left[\mathrm{~V} \mathrm{C}^{-1}\right]$ & Quantity & $k_{z, \text { total }}\left[\mathrm{V} \mathrm{pC}^{-1}\right]$ \\
\hline Resistive wall (Al) & $1.03 \times 10^{9}$ & $2200 \mathrm{~m}$ & 2.26 \\
\hline Pumping port (rf screen) & $3.65 \times 10^{2}$ & $2200 \mathrm{~m}$ & $8.04 \times 10^{-7}$ \\
\hline Connection part of MO flange & $1.28 \times 10^{7}$ & 2000 & 0.03 \\
\hline Comb-type bellows chamber & $3.00 \times 10^{9}$ & 1000 & 3 \\
\hline Gate valve & $3.00 \times 10^{9}$ & 30 & 0.09 \\
\hline SR mask & $1.82 \times 10^{-3}$ & 1000 & $1.82 \times 10^{-12}$ \\
\hline Vertical collimator & $3.37 \times 10^{10}$ & 4 & 0.15 \\
\hline Horizontal collimator & $4.34 \times 10^{10}$ & 7 & 0.3 \\
\hline Tapered beam pipe & $3.83 \times 10^{8}$ & 16 & $6.13 \times 10^{-3}$ \\
\hline beam position monitor (BPM) & $1.63 \times 10^{8}$ & 440 & 0.07 \\
\hline BPM for feedback & $5.90 \times 10^{8}$ & 10 & $5.90 \times 10^{-3}$ \\
\hline Transverse feedback kicker & $5.01 \times 10^{11}$ & 1 & 0.5 \\
\hline IP chamber & $8.00 \times 10^{8}$ & 1 & $8.00 \times 10^{-4}$ \\
\hline rf cavity (ARES) & $4.35 \times 10^{11}$ & 18 & 7.83 \\
\hline Total & & & 14.24 \\
\hline
\end{tabular}

collimator of KEKB. For instance, when the half aperture is $5 \mathrm{~mm}$ and the bunch length is $6 \mathrm{~mm}$, the loss factor in the SuperKEKB $\mathrm{f} 90 \times 220$ type horizontal collimator is approximately $0.043 \mathrm{~V} \mathrm{pC}^{-1}$, which corresponds to a power loss of $2.2 \mathrm{~kW}$ based on the design parameters of the LER (i.e., beam current of 3.6 A with 2500 bunches).

In the final configuration of the SuperKEKB LER, we intend to operate up to ten horizontal and four vertical collimators. When the half aperture of the horizontal and vertical collimators is set to 5 and $2 \mathrm{~mm}$, respectively, the total loss factor of the collimators is approximately $0.58 \mathrm{~V} \mathrm{pC}^{-1}$. The contribution of the LER to the total loss factor is approximately $4 \%$, as shown in Table $\mathrm{I}$, and this is also acceptable in terms of the rf system in SuperKEKB [16]. Then, longitudinal impedance models for the main ring have been constructed to study the longitudinal singlebunch microwave instabilities, and the simulations revealed that the bunch lengthening was less than $20 \%$ at the design bunch currents [17].

The kick factor in the vertical collimator should be rather large as it is necessary to close the aperture to the beam. This could induce TMCI and limit the bunch current. The threshold for the bunch current in the vertical direction, $I_{\text {th }}$, is expressed as

$$
I_{\mathrm{th}}=\frac{C f_{s} E}{\sum_{i} \beta_{y, i} k_{y, i}\left(\sigma_{z}\right)} .
$$

Here, $C, f_{s}, E, \beta_{y, i}, k_{y, i}$, and $\sigma_{z}$ are a constant $(\approx 8)$, the synchrotron frequency, beam energy, beta function in the vertical direction at component $i$, kick factor in the vertical direction at component $i$, and longitudinal bunch length, respectively [19]. The kick factor is a function of the bunch length. For the LER, the synchrotron frequency and bunch length are $2.47 \mathrm{kHz}$ and $6 \mathrm{~mm}$ in the design value, respectively. The bunch current should be smaller than this threshold to avoid TMCI.

Figure 9 shows the bunch current threshold as a function of the half aperture at D06V1 for two situations in the final optics configuration, where the beta function at the interaction point (IP) is $0.27 \mathrm{~mm}$, assuming a vertical emittance of $9.64 \mathrm{pm}$. The aperture of the beam pipe in QC1RP is $\pm 13.5 \mathrm{~mm}$, which corresponds to $85 \sigma_{y}\left(\sigma_{y}\right.$ is the standard deviation of the Gaussian-distributed beam in the vertical direction) with a beta function of $2890 \mathrm{~m}$. In this estimation, the aperture of the D02V1 collimator is set to $2.5 \mathrm{~mm}$, and the beta function is $111.75 \mathrm{~m}$. This aperture in D02V1 corresponds to $80 \sigma_{y}$, which is narrower than that in the QC1RP beam pipe; the collimator was operated with approximately this value of $\sigma_{y}$ in the 2019 Autumn run. It should be noted that the aperture of the D02V1 collimator was set to approximately $\pm 1.4 \mathrm{~mm}$ in the 2019 Autumn run because the beta function at the IP was $1 \mathrm{~mm}$, and the beta function at the D06V1 collimator is $14.6 \mathrm{~m}$ in this optics.

The designed bunch current in the LER is $1.44 \mathrm{~mA} \mathrm{bunch}^{-1}$ (3.6 A, 2500 bunches). When the aperture of the D03V1 collimator is opened to $\pm 9 \mathrm{~mm}\left(744 \sigma_{y}\right)$, the designed bunch current exceeds the threshold before the aperture of the D06V1 is closed to $80 \sigma_{y}$. When the D03V1 collimator is closed to $\pm 1 \mathrm{~mm}$, which is equivalent to $80 \sigma_{y}$, the design bunch current exceeds the threshold even though the D06V1 collimator is opened. Hence, we could not close the collimators aggressively, which may potentially pose a future problem for achieving a higher beam current with a low background signal. The discussion here is a rough 


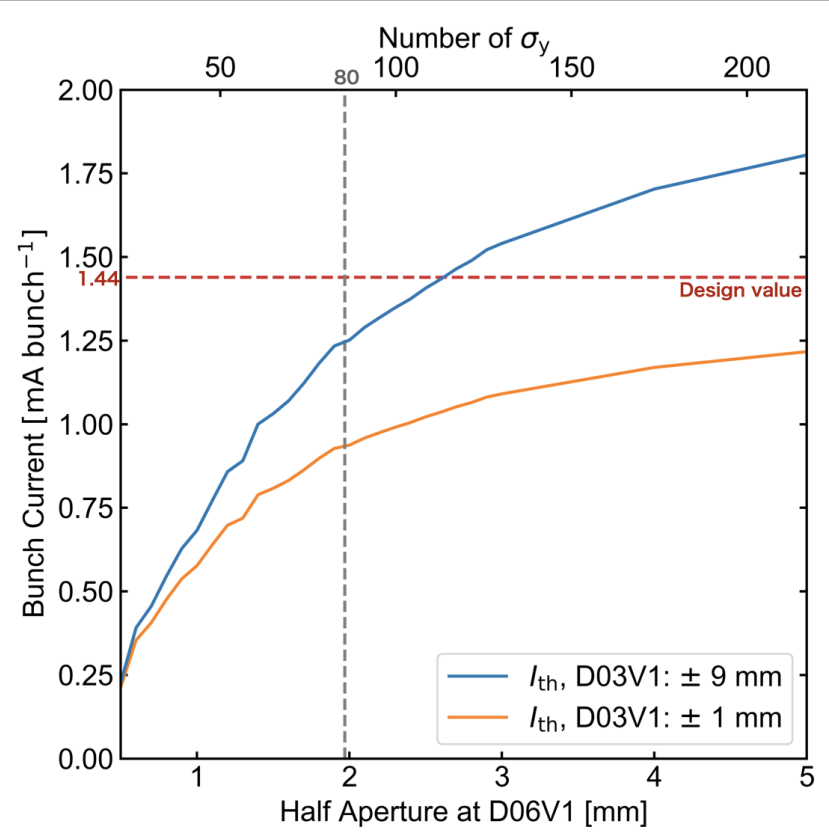

FIG. 9. Bunch current threshold owing to TMCI as a function of half aperture at D06V1 collimator in final optics configuration (beta function at IP: $0.27 \mathrm{~mm}$; vertical emittance: $0.64 \mathrm{pm}$ ). The transverse kick factor is calculated with GDFIDL. The vertical beta functions for the collimators are $111.75 \mathrm{~m}$ at D02V1, $16.96 \mathrm{~m}$ at $\mathrm{D} 03 \mathrm{~V} 1,19.24 \mathrm{~m}$ at $\mathrm{D} 06 \mathrm{~V} 2$, and $61.44 \mathrm{~m}$ at D06V1. The apertures in the D02V1 and D06V2 collimators are set to $\pm 2 \mathrm{~mm}\left(80 \sigma_{y}\right)$ and $\pm 9 \mathrm{~mm}\left(698 \sigma_{y}\right)$, respectively.

estimation based on the results from Eq. (1) and GDFIDL calculations; therefore, it is our intent to evaluate the TMCI using the actual beam in the coming commissioning as the present study is a rough estimation.

\section{Vacuum property}

The target pressure in the SuperKEKB main ring is the ultrahigh vacuum region where the static pressure is the order of $10^{-8} \mathrm{~Pa}$. The inner surface of the collimators is cleaned with acid to remove the oxide layer formed during machining. The ultrahigh vacuum in the collimator's chambers is maintained by pumping with sputter ion pumps and nonevaporable getter (NEG) pumps installed beside the collimators.

Prior to the 2019 Spring run in the phase- 3 commissioning, the collimators were installed without baking; however, the pressure near the collimators in the Tsukuba straight section had a notably raised background level in Belle II. Therefore, the collimators were baked in situ at approximately $100^{\circ} \mathrm{C}$ for $72 \mathrm{~h}$ using hot air after the run. Figure 10 shows the pressure normalized by the beam current; the pressure was measured with a cold cathode gauge (CCG), located near the D02H1 horizontal collimator. During the 2019 Autumn run, the $d P / d I$ was reduced by a factor of 5 compared to that during the Spring run because of the effect of the bakeout. Note that a water leak

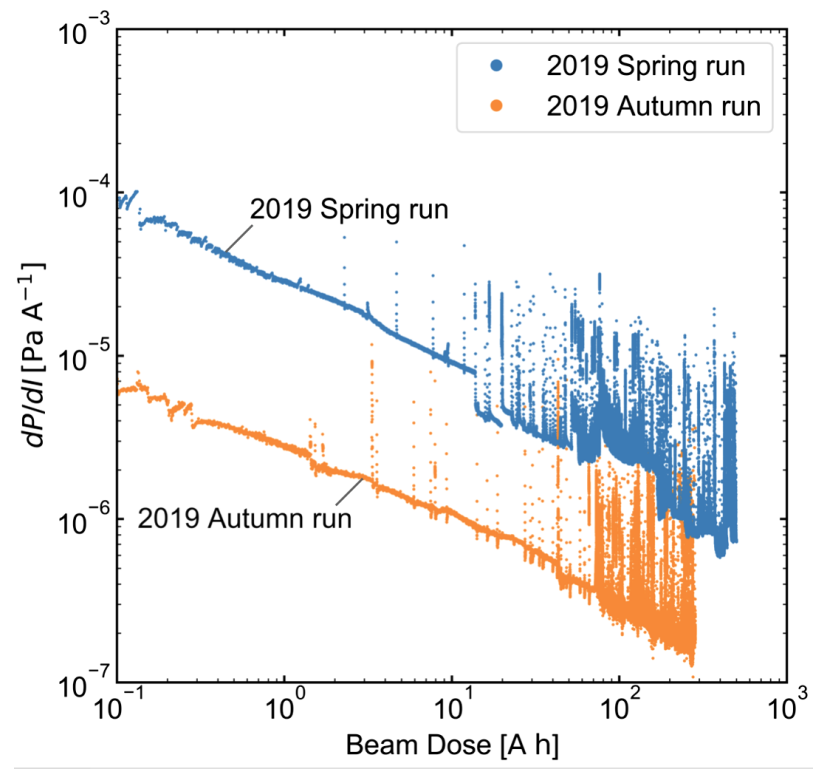

FIG. 10. Pressure-normalized beam current $(d P / d I)$ as a function of the beam dose, measured with CCG beside the D02H1 collimator.

from the cooling channel in one of the jaws to the vacuum chamber occurred after installation of this collimator before the Spring run due to a manufacturing defect; thus, the bakeout for this collimator was particularly effective.

\section{CONTROL SYSTEM}

Figure 11 shows a schematic of the configuration of the control system for the collimators; this configuration was installed in each control room. Programmable logic controllers (PLCs, FA-M3, Yokogawa Electric Corp.) control the collimators, and the Linux CPU module (F3RP71 or F3RP61, Yokogawa Electric Corp.) was used for the CPU. An experimental physics industrial control system (EPICS) was embedded in the CPU module [20].

In the control system for the KEKB-type collimators, positioning modules for the stepping motors, a digital output module to issue an emergency positioning stop request, a digital input module to detect the statuses of limit or home switches, and a general purpose interface bus module to measure the displacement of the jaw were embedded in the PLC, as shown in Fig. 11(a). The positioning module (F3NC01, Yokogawa Electric Corp.) controls two motors at most using one module. A potentiometer, which is a variable resistance instrument, measures the displacement of the jaw via the four-wire resistance measurement using a digital multimeter (DMM, 34870A/ 34902A, Keysight Technologies, Inc.) [21].

On the other hand, the PLC for the new collimators consists of the Linux CPU module and a positioning module (F3NC97, Yokogawa Electric Corp.), and the configuration is simplified compared with that of the conventional system, as shown in Fig. 11(b). The positioning 


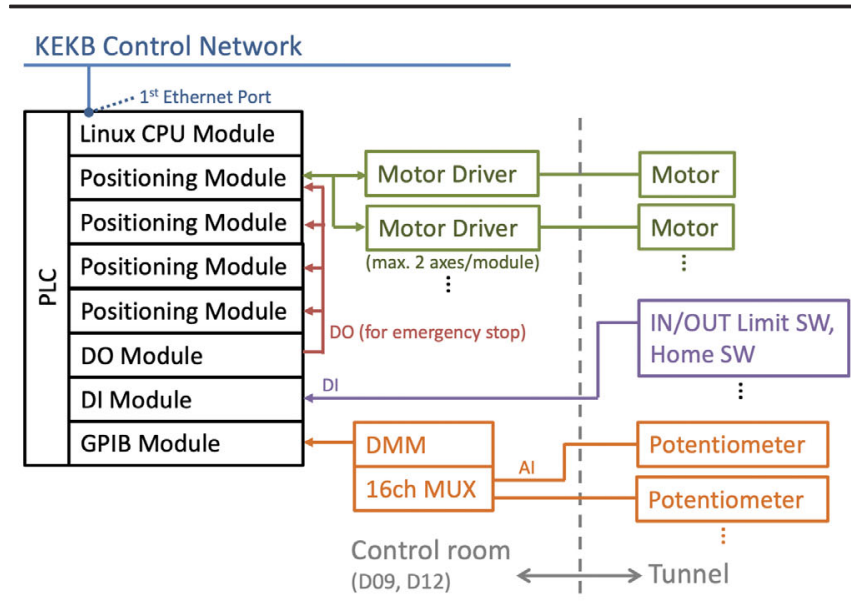

(a)

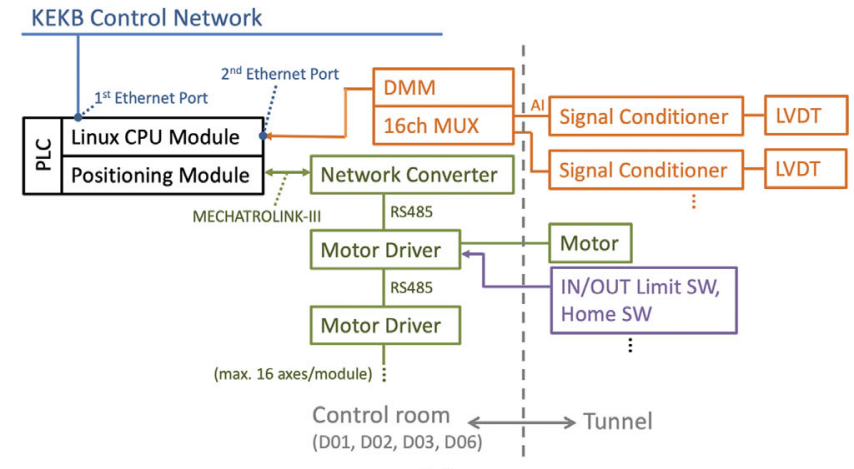

(b)

FIG. 11. Schematic configuration of control system for (a) KEKB-type collimators and (b) SuperKEKB-type collimators.

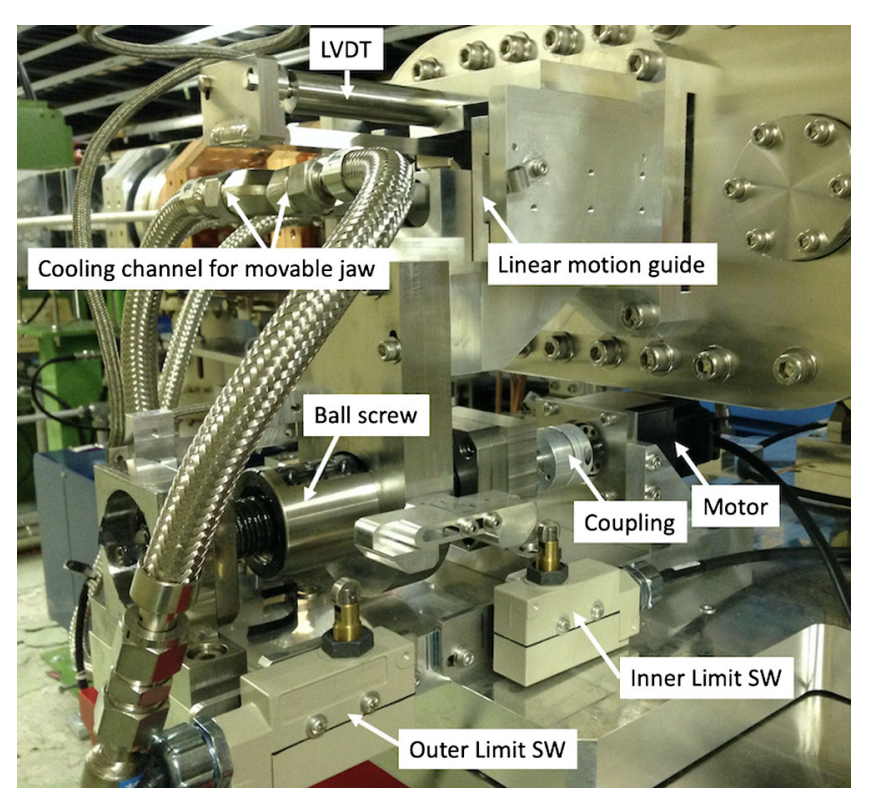

FIG. 12. Photograph of drive mechanism in the SuperKEKBtype collimators. module controls sixteen stepping motors using one module with a MECHATROLINK-III interface [22]. This configuration has high extensibility because the components in the underlayer of the system are packaged, which is an important feature as additional collimators are installed as the commissioning progresses. A DMM with an ethernet interface (34872A/34902A, Keysight Technologies, Inc.) is used to measure the displacement; it is directly connected to a second ethernet port of the Linux CPU module.

Figure 12 shows a photograph of the drive mechanism in the collimators. A linear variable differential transformer (LVDT, Model HCA2000-4019, Measurement SPECIALITIES Ltd.) for measuring the position of the jaw, two limit switches, and one home switch are used for one axis. The resolution of the displacement measurement is approximately $\pm 10 \mu \mathrm{m}$. A five-phase stepping motor (Model CRK564BKD-H50, Oriental Motors Ltd.) is used for the positioning. A no backlash ball screw (Model BIF2805M, THK Ltd.) was used in this setup; the motion of the jaw was set to $0.2 \mu \mathrm{m} \mathrm{step}^{-1}$ for the calculation. The bidirectional repeatability of the positioning is approximately $\pm 20 \mu \mathrm{m}$.

\section{PRESENT STATUS}

The newly installed collimators have been working up to the beam current of approximately $1 \mathrm{~A}$, generally with no problems. These collimators are indispensable for the operation of Belle II and SuperKEKB.

A typical example of the collimators used for background reduction is shown in Fig. 13. When the half aperture of the top side jaw in the D01V1 collimator was reduced from 3.15 to $2.50 \mathrm{~mm}$, the background measured with the diamond sensor of Belle II was reduced by approximately $20 \%$ (1 in Fig. 13). The background signal increased when an inside jaw, which is placed in the inner side of the ring in $\mathrm{D} 01 \mathrm{H} 4$, was closed, due to tip scattering (2 in Fig. 13).

Figure 14 shows the number of QCS quench occurrences by month. The collimators have been tuned since April $23 r d$ in 2018. Thereafter, the number of occurrences decreased dramatically; the collimator is very effective for preventing QCS quenches. The number of occurrences has decreased gradually with progress of the beam commissioning due to improved tuning of the collimators and optics.

Abnormal heating in the collimators themselves and in the components near them was not observed after installation of the collimators. Figure 15 shows the temperature profile when resistance temperature detectors (RTDs) were used for the D01H4 collimator and the bellows chambers right beside it. No abnormal temperature rise was observed with the RTD on the bellows, which is a connection between the movable jaw and the chamber in the collimator. This indicates that the rf shield around the jaw works well to prevent the HOM intrusion to the resonator structure 


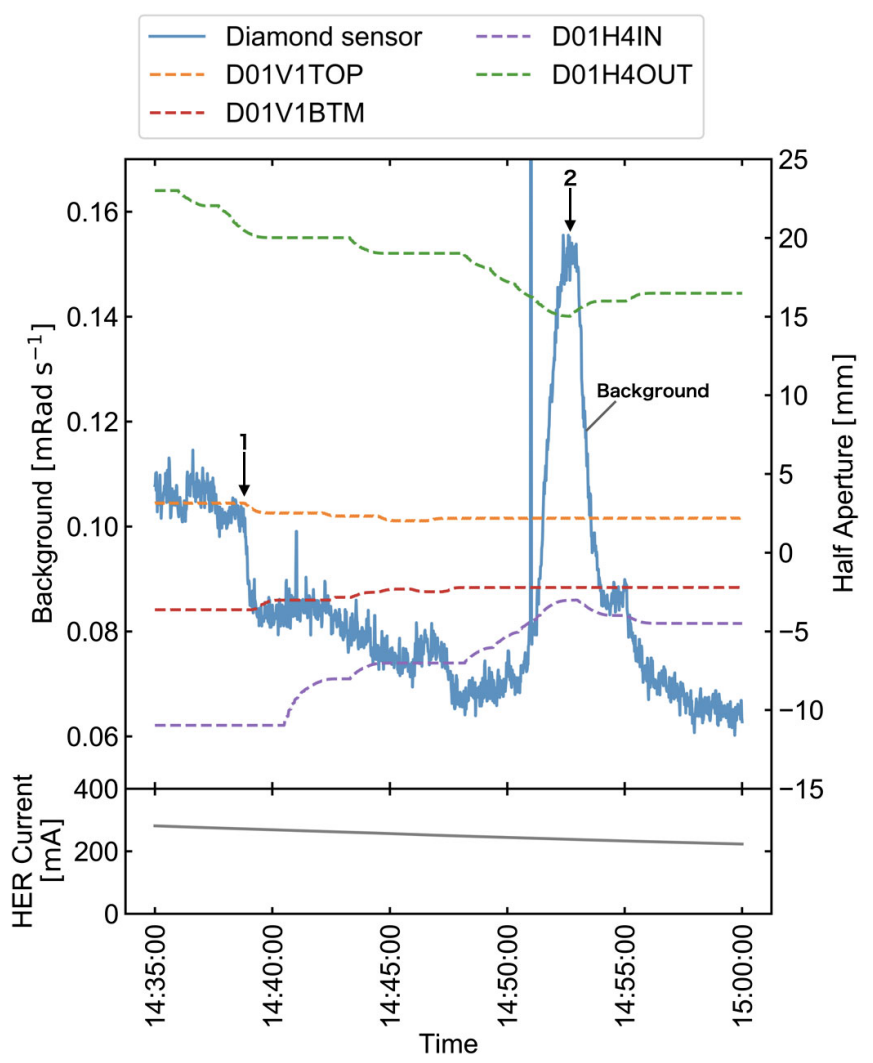

FIG. 13. Example of effect of collimators on background in a diamond sensor of Belle II; half aperture of $0 \mathrm{~mm}$ refers to the center of the beam channel.

behind the jaw. The temperature on the collimator and the bellows chambers beside it increased linearly. This heating is derived mainly from the SR, and no multipactor discharge occurred up to $940 \mathrm{~mA}$ with 1578 bunches.

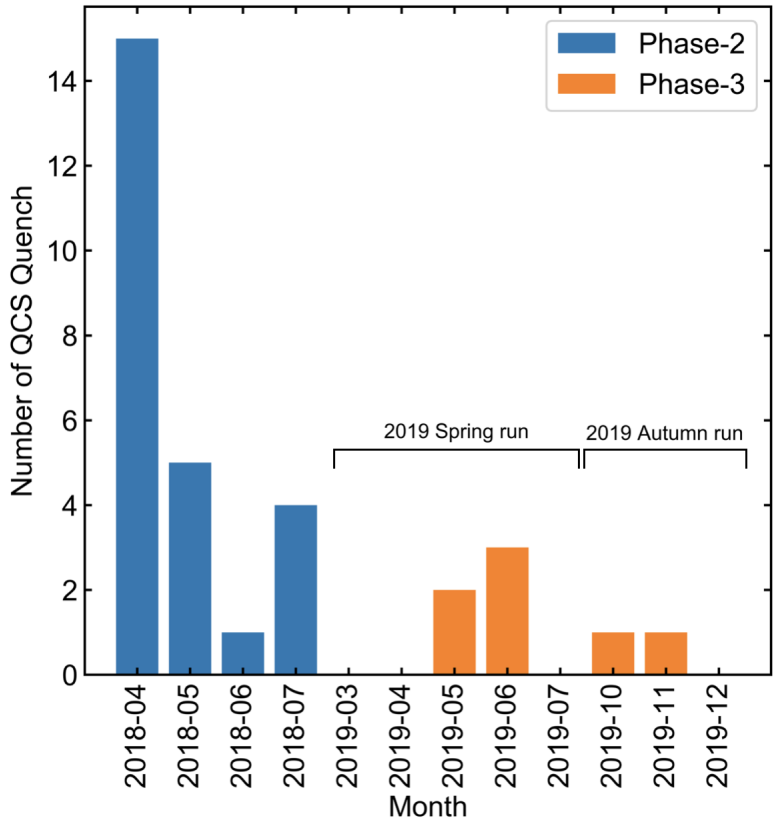

FIG. 14. Number of QCS quenches derived from beam loss.

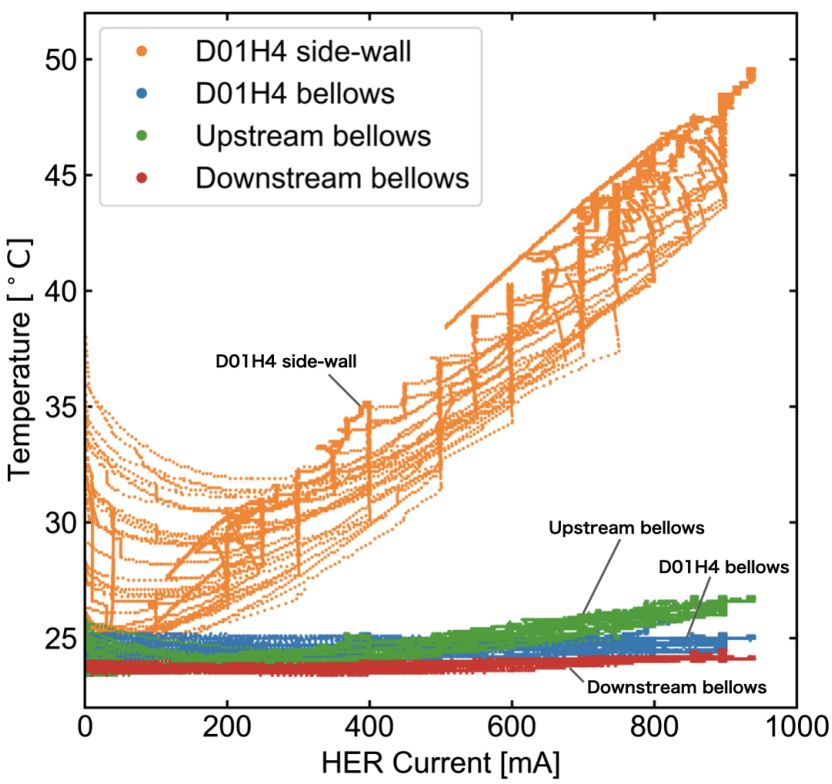

FIG. 15. Temperature on the D01H4 collimator and bellows chambers beside it, measured with RTDs, as a function of beam current in the HER. The flow rate of the cooling water was approximately $4 \mathrm{~L} \mathrm{~min}^{-1}$.

Note that the SR power density, which is derived from a bending magnet (bending radius: $147.9 \mathrm{~m}$ ) located approximately $6.8 \mathrm{~m}$ upstream of the $\mathrm{D} 01 \mathrm{H} 4$ on the tapered wall at an angle of $12^{\circ}$, is approximately $18.2 \mathrm{~kW} \mathrm{~m}^{-1}$ when the beam current is $2.6 \mathrm{~A}$; this is the highest power density in all of the collimators in SuperKEKB HER and LER.

In high-current operations at $500 \mathrm{~mA}$ or above, the jaws were occasionally damaged by hitting abnormal beams, as shown in Fig. 16. From approximately 1 RL onwards, a scar mark was observed on the tip of the top side jaw. Thereafter, a protrusion was formed by vapor deposition of tungsten because the half aperture had been set to very narrow $(1.5 \mathrm{~mm})$ during the 2019 Autumn run in this collimator. The damaged tungsten is likely to be crumbly owing to radiation embrittlement or recrystallization. When this problem occurs, the pressure measured using the CCGs near the collimator increases instantaneously, accompanied by a beam abort, as shown in Fig. 17. This issue occurs with low frequency, i.e., once per commissioning period currently but is a high-consequence issue because we are not able to apply high voltage on the detectors in Belle II, owing to high backgrounds, which hinders data acquisition. It takes at least 2-3 days to change the damaged jaws to new ones; therefore, the background had to be suppressed by shifting the collimator itself or making an orbit bump of approximately $1 \mathrm{~mm}$ in the horizontal direction to avoid the damaged region on the tips as a temporary solution during the commissioning period. The damaged jaw was changed during shutdown periods. We have not identified the cause of this phenomenon as no abnormalities, such as 


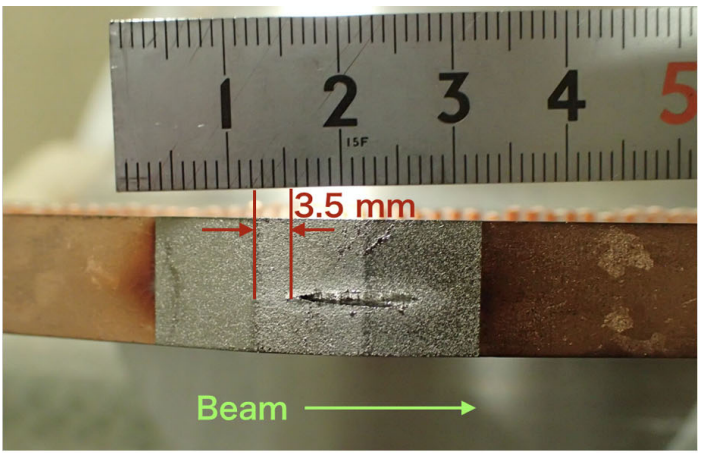

(a)

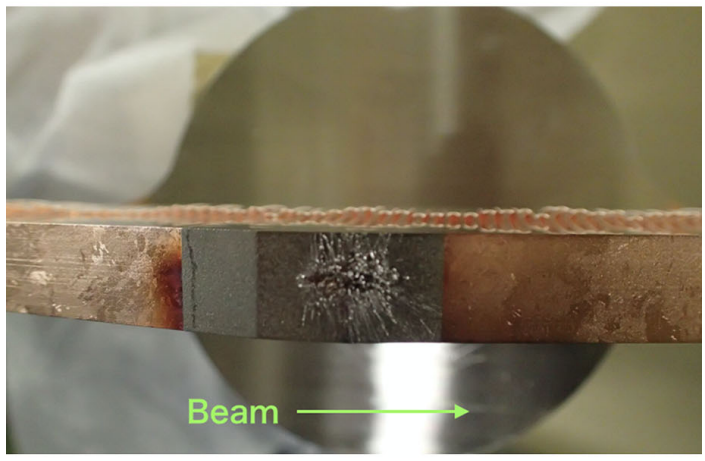

(b)

FIG. 16. Photograph of a damaged jaw in (a) a top side and (b) a bottom side of the D06V2 collimator. The traveling direction of the beam is from left to right in this photograph.

an abnormal beam orbit change, abnormal bunch oscillations, and so on, were observed before this problem arose.

The maximum temperature induced by the beam hit was estimated using FLUKA [23]. Figure 18 shows the maximum

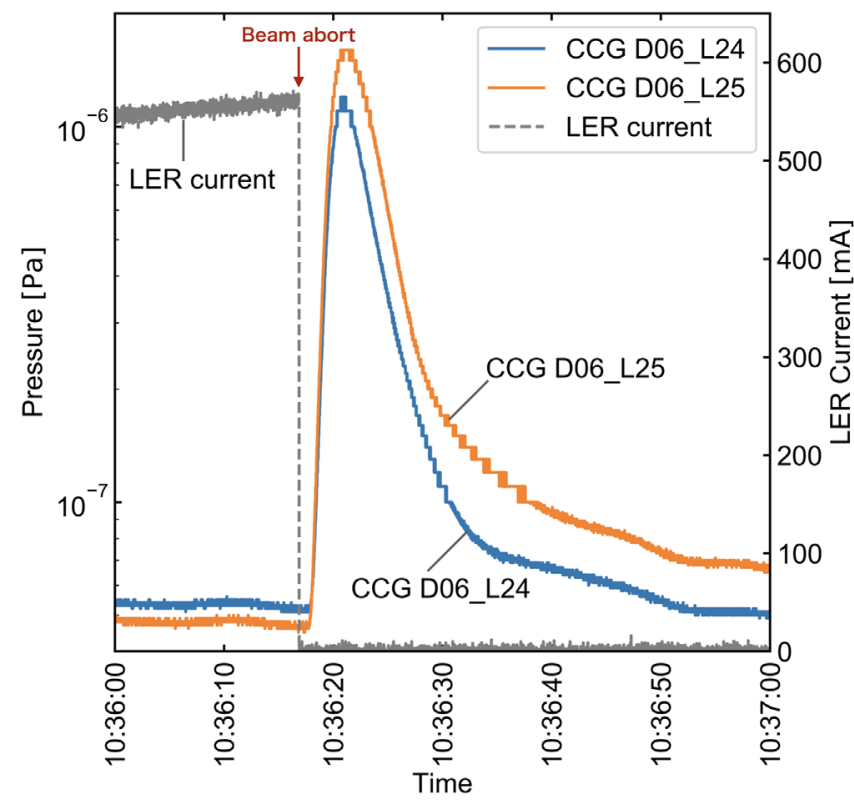

FIG. 17. Pressure burst in CCGs beside the D06V2 collimator and beam current in the LER. temperature along the path of an incident positron beam in the tungsten target for three different beam conditions. In this simulation, the tungsten target has a cylindrical shape with a length of $10 \mathrm{~mm}$ and diameter of $10 \mathrm{~mm}$; the $4 \mathrm{GeV}$ positron beam is injected into the center of the target. The beam profile is Gaussian, and the heat transfer is ignored here. Under any condition, the maximum temperature exceeds the melting point. However, the damage mark on the tip of the jaw was seen after approximately 1 RL (see Fig. 16); thus, the situation at the damage point would be equivalent to cases where the beam diameter is 0.1 or $0.5 \mathrm{~mm}$ (FWHM) in this simulation.

Materials with a short RL, such as tungsten, are very effective as beam halo shields; however, the energy deposit in the material is rather localized and the temperature of the material easily exceeds the melting point. In order to avoid damage to the jaw, we have started developing a collimator with low atomic number materials (low-Z). The low-Z collimator will be used to protect the collimators for background suppression. Figure 19 shows the maximum temperatures and melting points for each material. The beam profile is Gaussian, and the beam diameter (FWHM) and the induced current are $0.5 \mathrm{~mm}$ and $50 \mathrm{~mA}$, respectively. Under this condition, the maximum temperature does not exceed the melting point of titanium, aluminum and carbon (graphite) within $1 \mathrm{RL}$. The difference between the maximum temperature and the melting point is

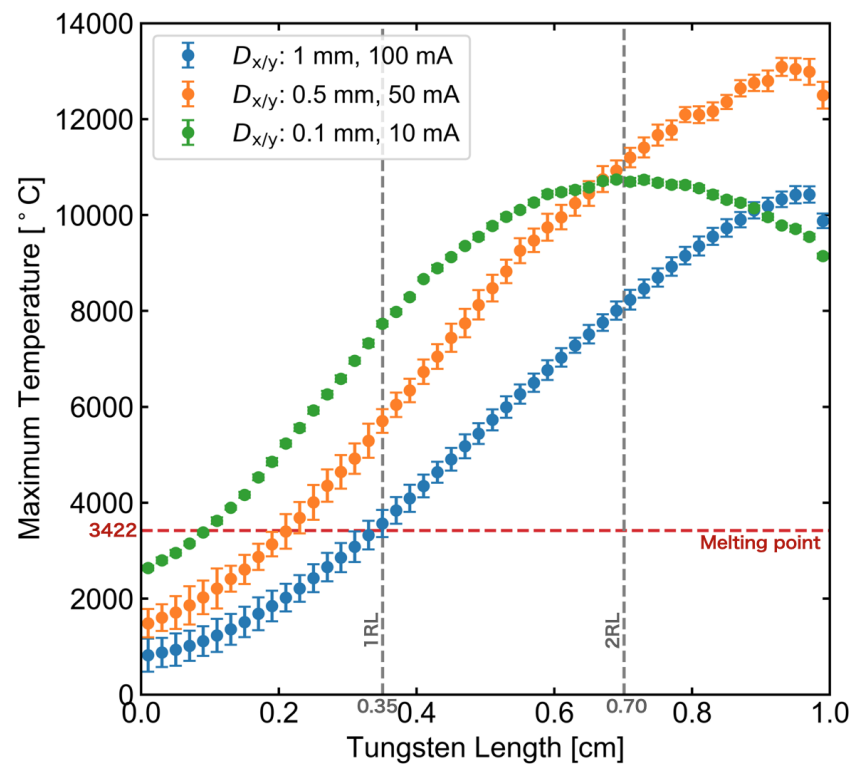

FIG. 18. Maximum temperature along the path of a positron beam in the cylindrical tungsten target. The beam profile and energy are respectively Gaussian and $4 \mathrm{GeV} . D_{x / y}$ refers to the diameter (FWHM) of the beam in the horizontal or vertical direction. The target has a length of $10 \mathrm{~mm}$ and diameter of $10 \mathrm{~mm}$. The environment around the target is set to vacuum. The revolution frequency in the main ring is $100 \mathrm{kHz}$, and the beam current of $10 \mathrm{~mA}$ corresponds to approximately $6.24 \times 10^{11}$ positrons, for example. 


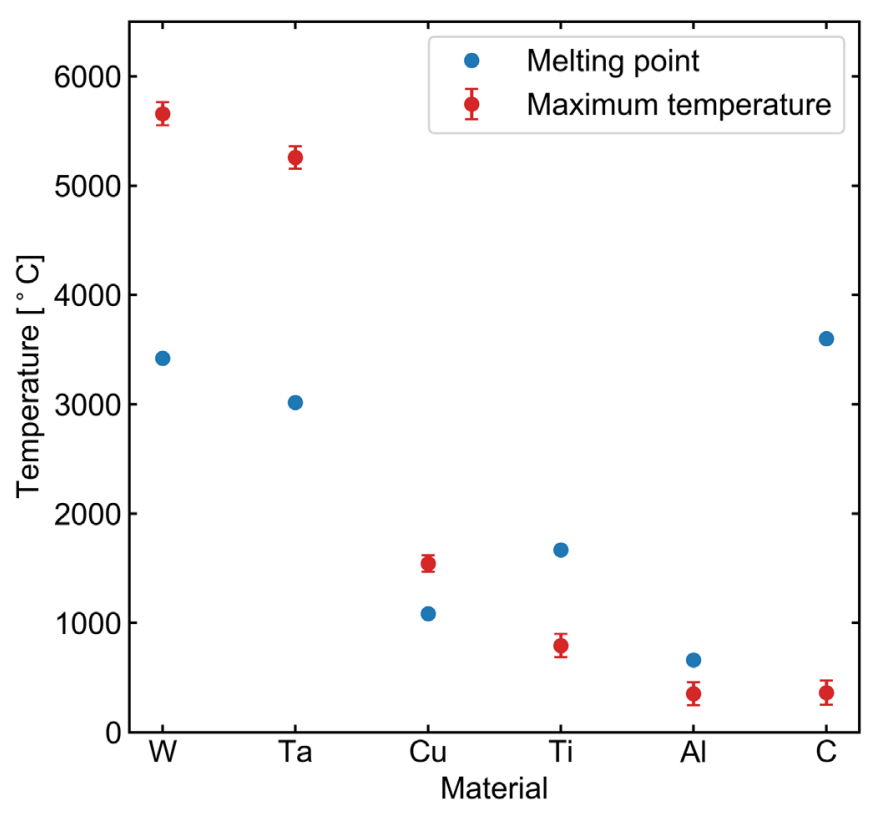

FIG. 19. Maximum temperatures within $1 \mathrm{RL}$ and melting points for each material, induced by the positron beam. The beam profile and energy are respectively Gaussian and $4 \mathrm{GeV} . D_{x / y}$ and the injected current are $0.5 \mathrm{~mm}$ and $50 \mathrm{~mA}\left(3.12 \times 10^{12}\right.$ positrons), respectively. The target has a length of $2 \mathrm{RL}$ and diameter of $10 \mathrm{~mm}$. The environment around the target is set to vacuum. The RL is $0.35 \mathrm{~cm}$ for tungsten, $0.41 \mathrm{~cm}$ for tantalum, $1.46 \mathrm{~cm}$ for copper, $3.56 \mathrm{~cm}$ for titanium, $8.90 \mathrm{~cm}$ for aluminum, and $19.32 \mathrm{~cm}$ for carbon.

approximately $875^{\circ} \mathrm{C}$ for titanium, $307^{\circ} \mathrm{C}$ for aluminum, and $3236^{\circ} \mathrm{C}$ for carbon; therefore, the titanium and carbon are hard to break. Because titanium and carbon have been successfully used as collimator materials in KEKB and LHC [24], respectively, these materials are candidates for the low-Z collimator in SuperKEKB.

\section{SUMMARY AND FUTURE PLANS}

The newly developed collimators for the main ring of SuperKEKB are now working well with few issues up to a beam current of approximately $1 \mathrm{~A}$. Investigation of the soundness of these collimators at higher beam current is ongoing.

The damaged tungsten tips become brittle when the jaws are damaged by contact with the beams. Therefore, we developed a jaw which has a tantalum tip to avoid the embrittlement. Damage to the tip occurs from approximately 1 RL onwards; hence, a jaw with a shorter length tantalum tip of $5 \mathrm{~mm}$ was developed to mitigate the energy deposit derived from the particle shower. The performance declines when the non-Gaussian tail shield with this shorter tip is used; thus, this collimator (D06V1) was installed in the arc section far from the IP, as shown in Fig. 2. It is our intent to test the shorter tip in the 2020 Spring commissioning.
Development of a low-Z collimator is in progress, with the aim to avoid damage to the collimators for background suppression. The candidate materials for the low-Z collimator are titanium and carbon, and we intend to manufacture a testing jaw and install it on one side of an existing collimator in the LER during the 2020 Summer or Winter shutdown period.

\section{ACKNOWLEDGMENTS}

We thank all the staff of the SuperKEKB accelerator and Belle II detector for their cooperation.

[1] Y. Ohnishi et al., Accelerator design at SuperKEKB, Prog. Theor. Exp. Phys. 2013, 03A011 (2013).

[2] Y. Suetsugu, K. Kanazawa, K. Shibata, T. Ishibashi, H. Hisamatsu, M. Shirai, and S. Terui, Design and construction of the SuperKEKB vacuum system, J. Vac. Sci. Technol. A 30, 031602 (2012).

[3] E. Kou et al., The Belle II physics book, Prog. Theor. Exp. Phys. 2019, 123C01 (2019).

[4] N. Ohuchi et al., Final-focus superconducting magnets for SuperKEKB, in Proceedings of the International Particle Accelerator Conference, Vancouver, BC, Canada, 2018 (JACoW, CERN, Geneva, 2018), pp. 1215-1219, https:// doi.org/10.18429/JACoW-IPAC2018-TUZGBE2.

[5] A. Morita, Status of early SuperKEKB phase-3 commissioning, in Proceedings of the International Particle Accelerator Conference, Melbourne, Australia, 2019 (JACoW, CERN, Geneva, 2019), pp. 2255-2257, https:// doi.org/10.18429/JACoW-IPAC2019-WEYYPLM1.

[6] Y. Suetsugu, T. Kageyama, K. Shibata, and T. Sanami, Latest movable mask system for KEKB, Nucl. Instrum. Methods Phys. Res., Sect. A 513, 465 (2003).

[7] D. Zhou, K. Ohmi, and A. W. Chao, Beam tilt due to transverse wakefields for DA $\Phi$ NE, SuperB, KEKB and SuperKEKB, in Proceedings of the International Particle Accelerator Conference, San Sebastian, Spain, 2011 (JACoW, CERN, Geneva, 2011), pp. 601-603.

[8] H. Nakayama et al., Small-beta collimation at SuperKEKB to stop beam-gas scattered particles and to avoid transverse mode coupling instability, in Proceedings of the International Particle Accelerator Conference, New Orleans, Louisiana, 2012 (JACoW, CERN, Geneva, 2012), pp. 1104-1106.

[9] Y. Suetsugu, K. Kanazawa, K. Shibata, T. Ishibashi, H. Hisamatsu, M. Shirai, and S. Terui, Results and problems in the construction phase of the SuperKEKB vacuum system, J. Vac. Sci. Technol. A 34, 021605 (2016).

[10] Y. Suetsugu, K. Kanazawa, K. Shibata, M. Shirai, A. E. Bondar, V. S. Kuzminykh, A. I. Gorbovsky, K. Sonderegger, M. Morii, and K. Kawada, Development of bellows and gate valves with a comb-type rf shield for high-current accelerators: Four-year beam test at KEK B-Factory, Rev. Sci. Instrum. 78, 043302 (2007).

[11] I. Zagorodnov and K. L. F. Bane, Wakefield calculations for 3D collimators, Stanford Linear Accelerator Center (SLAC) Report No. SLAC-PUB-11938. 
[12] S. DeBarger et al., The PEP-II movable collimators, Stanford Linear Accelerator Center (SLAC) Report No. SLAC-PUB-11752.

[13] J. T. Seeman, Last year of PEP-II B-factory operation, in Proceedings of the European Particle Accelerator Conference, Genoa, Italy, 2008 (JACoW, CERN, Geneva, 2008), pp. 946-950.

[14] CST STUDIO Suite, https://www.3ds.com/products-services/ simulia/products/cst-studio-suite/?utm_source=cst.com\& utm_medium $=301 \& u t m \_$campaign $=$cst.

[15] The GDFIDL electromagnetic field simulator, http://www .gdfidl.de.

[16] K. Akai, RF overview, The 15th KEKB Accelerator Review Committee, http://www-kekb.kek.jp/MAC/2010/ Report/Akai_RF.pdf.

[17] D. Zhou et al., Impedance calculation and simulation of microwave instability for the main rings of SuperKEKB, in Proceedings of the International Particle Accelerator Conference, Dresden, Germany, 2014 (JACoW, CERN,
Geneva, 2014), pp. 1600-1602, https://doi.org/10.18429/ JACoW-IPAC2014-TUPRI021.

[18] K. Shibata, Estimation of impedances and loss factors of SuperKEKB LER, The 15th KEKB Accelerator Review Committee, http://www-kekb.kek.jp/MAC/2010/Report/ Shibata.pdf.

[19] Handbook of Accelerator Physics and Engineering (World Scientific, Singapore, 2009).

[20] EPICS home at Argonne, https://epics.anl.gov.

[21] Electrical Measurement, Signal Processing, and Displays (CRC Press, Florida, 2003).

[22] MECHATROLINK-III Features, https://www.mechatrolink .org/en/mechatrolink/feature-m3.html.

[23] FLUKA, http://www.fluka.org/fluka.php.

[24] A. Bertarelli et al., Mechanical design for robustness of the LHC collimators, in Proceedings of the Particle Accelerator Conference, Knoxville, Tennessee, 2005 (JACoW, CERN, Geneva, 2005), pp. 913-915, https://doi.org/ 10.1109/PAC.2005.1590608. 